\title{
Tecnica alternativa para prevenir la estimulación del nervio obturador durante la RTU vesical de neoformaciones en cara lateral
}

\author{
J. García Rodríguez*, A. Jalón Monzón, R. Carlos González Álvarez, C. Ardura Laso**,
}

J. M. Fernández Gomez, J.J. Rodríguez Martínez, F. J. Martínez Gómez, J. Regadera Sejas, F. González Huergo*

*Servicio Urologia. Hospital Comarcal de Jarrio. Coaña. Asturias. Servicio de Urologia I. Hospital Central de Asturias. Oviedo. Asturias. ${ }^{*}$ Servicio Anestesia y Reanimacion. Hospital Central De Asturias. Oviedo. Asturias.

Actas Urol Esp 2005; 29 (5): 445-447

\section{RESUMEN}

TECNICA ALTERNATIVA PARA PREVENIR LA ESTIMULACION DEL NERVIO OBTURADOR DURANTE LA RTU VESICAL DE NEOFORMACIONES EN CARA LATERAL

Objetivo: Presentamos una alternativa de abordaje para el bloqueo del nervio obturador durante la RTU vesical.

Material y Metodos: Se revisan retrospectivamente 400 tumores vesicales intervenidos, correspondientes a 218 pacientes, localizándose un $46,8 \%$ de estos en caras laterales. Se utiliza una vía de abordaje alternativa para el bloqueo del nervio obturador.

Resultados: Mediante este abordaje, que simplifica, en posición de litotomía, el bloqueo nervioso del obturador, conseguimos en más del 95\% de resecciones el que no se produzca estimulación muscular.

Conclusiones: Aplicando esta técnica, disminuimos el riesgo de perforación vesical por esta causa, evitando las complicaciones asociadas.

Palabras clave: Nervio obturador. RTU vesical.

\section{ABSTRACT}

AN ALTERNATIVE TECHNIQUE TO PREVENT OF OBTURATOR NERVE STIMULATION DURING LATERAL BLADDER TUMOURS TRANSURETHRAL RESECTION

Objetives: We presents an alternative to prevent the obturator nerve stimulation during TUBR.

Material and Method: We revise 400 bladder tumours corresponding to 218 patients, 46,8\% of them localized in lateral bladder wall. An alternative technique is used for that blockade.

Results: There have been no adductor contractions in more than 95\% of TUBR.

Conclusions: The alternative technique may reduce the stimulation of the obturador nerve and the risk of bladder perforation.

Keywords: Obturator nerve. TUBR.

$\mathrm{C}$ omo sabemos, la resección transuretral (RTU) es el tratamiento de elección en los tumores superficiales vesicales.

Entre las complicaciones que se pueden presentar, la estimulación del nervio obturador por el asa de diatermia en resecciones sobre cara lateral de la vejiga, podría causar perforación iatrogénica de esta, debido a la contracción del músculo aductor.
Presentamos una alternativa para el bloqueo del nervio obturador, con el paciente en posición de litotomía forzada, de fácil y rápida ejecución por el urólogo y que permite evitar este riesgo.

\section{MATERIAL Y RESULTADOS}

Se revisan retrospectivamente 400 tumores vesicales intervenidos, correspondientes a 218 pacientes, el $46,8 \%$ de estos se sitúan en las caras 
laterales de la vejiga, es decir casi la mitad de los pacientes con tumor vesical podrian ser subsidiarios de esta técnica.

Desde que lo realizamos, todos los pacientes que presentan tumor en cara lateral, porción inferolateral, son bloqueados por sistema en función de la preferencia del cirujano, previo o durante la RTU.

Con el paciente situado en la mesa de quirófano y en posición de litotomía forzada, tras realizar la anestesia raquídea, a $2 \mathrm{~cm}$ caudal y lateral al tubérculo pubiano, abarcaremos entre los dedos índice y pulgar el aductor mayor, introduciendo una aguja espinal unipolar de $3 / 80$ 22Gx80 mm ó de 3/120 20Gx120 mm para realizar la técnica. La aguja, conectada a un neuroestimulador, progresará en dirección craneallateral por el borde inferior del músculo. Localizamos así el nervio utilizando un neuroestimulador tipo Stimulator LA Type 861 Pajunk. Una vez localizado el nervio, este se bloquea con una ampolla de $10 \mathrm{cc}$. de mepivacaína clorhidrato al $1 \%$ sin vasoconstrictor, si fuese necesario podríamos utilizar hasta 4 ampollas al $1 \%$ ó 2 al $2 \%$. (Fig 1). Logramos en más del $95 \%$ de pacientes el bloqueo completo del nervio. En ocasiones sobretodo en pacientes obesos no conseguimos localizar el nervio y en otras observamos que a pesar de estimularlo e inyectar el anestésico, el paciente presenta igualmente contracción muscular, quizás se deba a infiltraciones distales a la

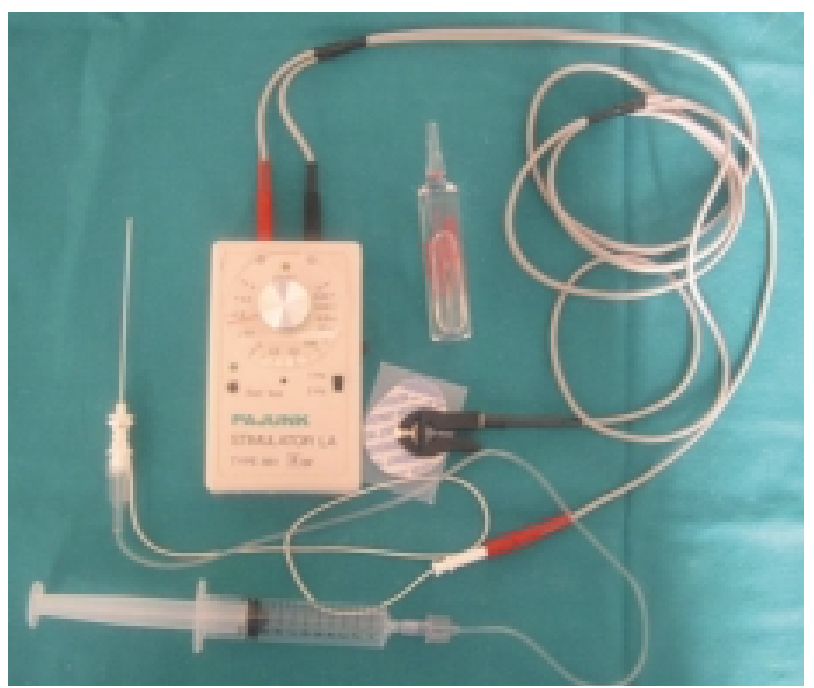

FIG 1. Neuroestimulador tipo Stimulator LA Type 861 Pajunk. zona estimulada. En ningún paciente hemos detectado efectos sistémicos secundarios al anestésico tras realizar la técnica.

\section{DISCUSIÓN}

El nervio obturador nace del plexo lumbar, por tres raíces procedentes de los pares lumbares $2^{\circ}$, $3^{\circ}$ y $4^{\circ}$, sigue un trayecto cercano a la porción inferolateral de la vejiga, cuello vesical y lateral a uretra prostática. Continua en profundidad por el canal obturador dividiéndose en dos ramas, una anterior, para el aductor menor, mediano y recto interno, y otra posterior para el aductor mayor, obturador externo y ramas articulares ${ }^{1}$.

Durante la RTU vesical, la estimulación del nervio obturador por el asa de diatermia en resecciones sobre cara lateral de la vejiga, podría causar perforación iatrogénica de esta, debido a la violenta contracción del músculo aductor.

Las técnicas clásicas para evitar la estimulación del nervio son varias, las mas comúnmente utilizadas son entre otras, el bloqueo de la transmisión neuromuscular mediante agentes como D-tubocuranina y succinilcolina, realizar la RTU con la vejiga a media repleción disminuyendo el voltaje de la electrocoagulación, colocación de la placa de electrodo indiferente en el lado contralateral a la lesión ${ }^{2}$, el uso de la coagulación para la resección de los chips de tejido o realizar cortes pequeños con el asa de diatermia. Últimamente la utilización de resectores de alta frecuencia ${ }^{3}$ y por supuesto las técnicas de bloqueo nervioso ${ }^{4-6}$, incluidas las realizadas por vía endoscópica transparietal ${ }^{7}$.

Augspurger describe en 1980 la técnica clásica para el bloqueo. Con el paciente en decúbito supino, se realiza una marca $1-2 \mathrm{~cm}$ lateral y 1$2 \mathrm{~cm}$ caudal al tubérculo pubiano y se hace avanzar una aguja $22 \mathrm{~g}$ de $10 \mathrm{~cm}$ perpendicular con dirección ligeramente medial hacia el canal obturador. El nervio obturador está localizado $2-3 \mathrm{~cm}$ pasado el punto inicial de contacto con el ramo pubiano. En este punto(A) se inyecta un tercio del anestésico, los dos tercios restantes a un centímetro a cada lado de este punto $(\mathrm{B}, \mathrm{C})^{8}$ (Fig. 2).

Describimos una vía de abordaje alternativa para el bloqueo del nervio obturador, utilizando un neuroestimulador y con el paciente en posición de litotomía forzada. (Figs. 3 y 4). 


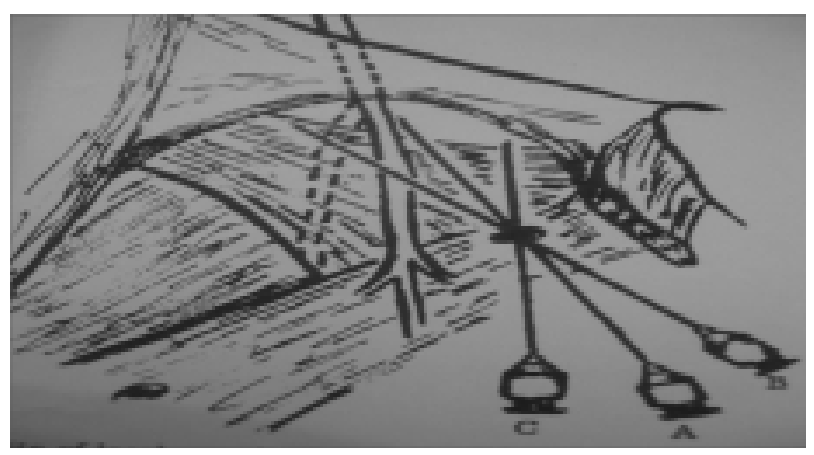

FIG 2. Técnica clásica según Augspurger ( Ilustración "Prevention of obturator nerve stimulation during transurethral surgery".J Urol,1980).

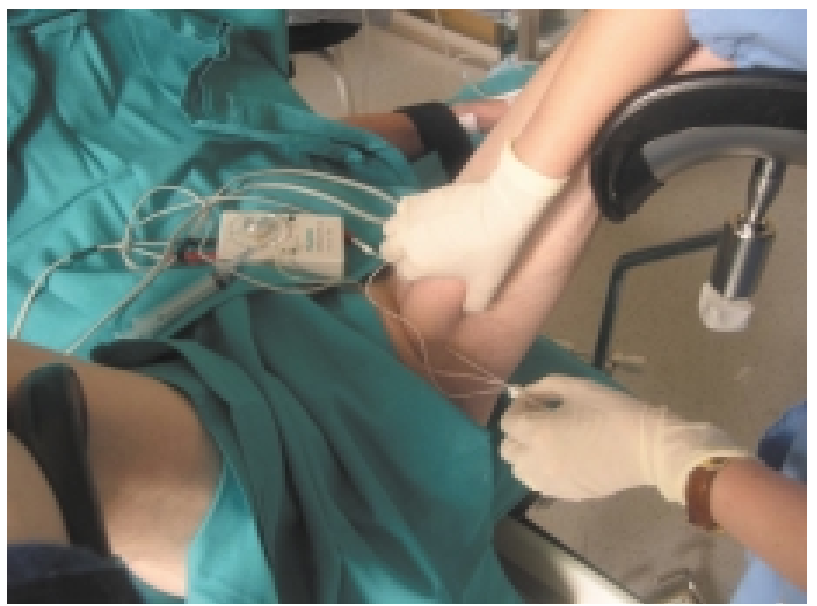

FIG 3. Técnica de bloqueo en posición de litotomía.

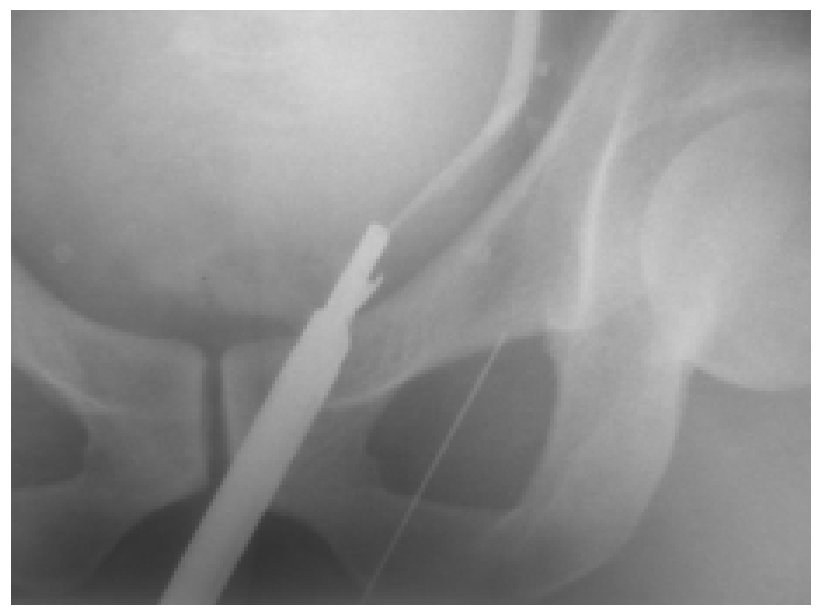

FIG 4. Dirección de aguja.
El área a infiltrar coincide con el punto de contracción muscular, que objetivaremos claramente en la mayoría de las ocasiones. Una vez localizado el nervio se bloquea con Mepivacaína Clorhidrato al $1 \%$.

Mediante este nuevo abordaje, que simplifica en posición de litotomía, el bloqueo, conseguimos en más del $95 \%$ de resecciones el que no se produzca estimulación muscular del aductor. Concluimos al igual que otros autores ${ }^{9}$ que con el bloqueo disminuye el riesgo de perforación vesical, evitando así las complicaciones asociadas, y permitiéndonos a la vez trabajar con mucha más seguridad.

\section{REFERENCIAS}

1. L. Testut. Tratado de anatomía humana. Sexta Edición. Edit Salvat. Tomo tercero. Capitulo II: 153-289.

2. Kihl B, Nilson AE, Pettersson S. Thig adductor contraction during transurethral resection of bladder tumours: evaluation of inactive electrode placement and obturator nerve topography. Scand J Urol Nephrol 1981;15:121-125.

3. Shiozawa H, Aizawa T, Ito T, Miki M. A new transurethral resection system: operating in saline enviroment precludes obturator nerve reflexes. J Urol 2002;168:2665-2667.

4. Gasparich JP, Mason JT, Berger RE. Use of nerve stimulator for simple and accurate obturator nerve block before transurethral resection. J Urol 1984;132:291-293.

5. Hradec E, Soukup F, Novak J, Bures E. The obturator nerve block. Preventing damage of the bladder wall during transurethral surgery. Int Urol Nephrol 1983;15:149-153.

6. Hoffmann P, Meyer O. Blockade of obturador reflex. Anaesthesist 1980;29:55-56

7. Zuluaga Gómez A, Torres Ramírez C, Martínez Torres JL, de la Fuente Serrano A, Abad Menor F, Montero Jara V. Inhibición de la estimulación del nervio obturador durante la cirugía transuretral. "Bloqueo endoscópico transparietal" Actas Urol Esp 1983;7:321-324.

8. Augspurger RR, Donohue RE. Prevention of obturator nerve stimulation during transurethral surgery. J Urol 1980;123: 170-172.

9. Delliveliotis C, Alexopoulou K, Picramenos D, Econornacos G, Goulandris N, Kostakopoulos A. The contribution of the obturator nerve block in transurethral resection of bladder tumors. Acta Urol Belg 1995;63:51-54.

Dr. J. García Rodríguez

C/ Martínez Vigil, 2- 2o dcha

33010. Oviedo (Asturias)

(Trabajo recibido el 21 noviembre de 2005) 